\title{
THE EFFECT OF THE HOT, ANISOTROPIC MAGNETOSPHERIC PROTONS ON THE DISPERSION RELATION
}

\author{
K. V. Gamayunov,* G. V. Khazanov,** A. A. Veryaev* and \\ T. I. Gombosi** \\ * Altai State University, Barnaul 656099, Russia \\ ** Space Physics Research Laboratory, Department of Atmospheric, Oceanic \\ and Space Science, University of Michigan, Ann Arbor, MI 48109-2143, \\ U.S.A.
}

\begin{abstract}
It is shown that the presence of hot, anisotropic protons of ring current in outer plasmasphere lead to the modification of the dispersion of A- and FMS-waves in the low frequency region. In this case, a periodic instability of A-waves is observed, and during their propagation, the change of polarization is possible. This can explain the presence of right-hand polarization waves in the ring current zone.
\end{abstract}

\section{INTRODUCTION}

It is common knowledge that the ring current has a significant effect on the dynamics of ionosphere-magnetosphere interactions by generating MHD waves. In this connection, while describing interactions of waves with plasma particles, there arises the need to know the spectral energy density of Alfven (A) and fast magnetosonic (FMS) waves.

In the satellite measurements, carried out in the overlap zone of the ring current and the plasmasphere, fairly insensitive electromagnetic waves with frequencies of $0.1-10 \mathrm{~Hz}$ were detected (see e.g. $2,10,17 /$ and references therein). These waves are identified as ICW's and are observed in an approximately equal mixture of left-hand polarized ( $\mathrm{L}$ or $\mathrm{A}$ ) and right-hand polarized $(\mathrm{R}$ or FMS ) modes. The source of the free energy for the generations of ICW's is the hot ions with anisotropic temperature $\left(T_{\perp}>T_{\|}\right)$. However, the condition for generation is fulfilled only for Lwaves (see $/ 3,8 /$ and others). In this connection, there arises a question of mechanisms of emergence of the observed R-waves.

For a long time the ICW's propagating along the external magnetic field were studied only in ground experiments $/ 5,7 /$ and only about 15 years ago they began to be studied intensively using satellites. The first direct measurements of these waves were carried out on ATS-1 /2/. GEOS-1 and GEOS-2 researchers continued their study of these waves using special instrumentation on board the satellites intended for investigation oscillations in the region of $0.1-10 \mathrm{~Hz} / 5,9,14,16,17 /$. These experiments revealed a substantial effects of helium ions, the presence of magnetic fields $\delta B$ with $L-$ and R-polarization's, were detected. Similar results were also obtained on ACE-6 /4,12,13/. Experimental detection of the importance of helium ions in generation and propagation of ICW's gave rise to a series of theoretical papers. In these papers the dispersive properties of a plasma in the presence of heavy ions (see e.g. /11/ and others), as well as ray trajectories of ICW's with the presence of an addition of helium ions in the plasma (see e.g. $/ 15,17 /$ ), were calculated. One of the principle results of these works is the possibility of liner transformation in an inhomogeneous plasma of lefthand polarized waves into right-hand polarized waves in the presence of helium ions $19,17 /$. Thus, at present, the generally accepted mechanism of the emergence of the R-mode in the overlap region of the plasmasphere and the ring current is the following: L-polarized waves are generated in the equatorial region at the expense of anisotropy of ring current protons $\left(T_{\perp}>T_{\| 1}\right)$ and propagate into the region of higher magnetic latitude. If their frequency is greater than the equatorial crossover frequency, then propagating in the inhomogeneous plasma and reaching the local crossover 
frequency, they transfrorm into right-hand polarized oscillations. From this discussion it follows that the frequency of oscillations with the R-polarization registered on GEOS-1, must be greater than the equatorial crossover frequency. However, in some cases R- oscillations with a lower frequency were registered.

In the present paper we will consider the change of the dispersive properties of MHD oscillations in the presence of a small addition of hot anisotropic protons. Taking into account the group of hot anisotropic ions leads, in particular, to the possibility of transformation of L- polarized oscillations into $R$ - polarized waves during the propagation in an inhomogneous plasma. This effect can be of substantial importance in magnetospheric conditions because there are satellite measurement in which both $\mathbf{L}$ - and $\mathbf{R}$ - polarized waves with frequencies substantially lower than the local helium gyrofrequency, were registered (see e.g. /10/). Unfortunately, from the results obtained on the IRM satellite, it is impossible to say anything about the effect of helium ions upon the registered ICW's, since no simultaneous observations of ICW's and the ion composition of plasma were carried out.

\section{MHD-OSCILLATIONS IN THE PRESENCE OF A SMALL ADDITION OF HOT ANISOTROPIC PROTONS}

Let us consider the electron-proton plasma placed in a homogeneous field $\vec{B}_{0}$. Assuming the main part of particles to be cold, let us write the dispersive equation for A- and FMS- waves. In the coordinate system with axis $\mathrm{z}$ along the extemal magnetic field, we have /1/:

$$
\frac{c^{2} k^{2}}{\omega^{2}} \equiv N^{2}=\frac{1}{2 \cos ^{2} \theta}\left[\varepsilon_{1}\left(1+\cos ^{2} \theta\right) \pm \sqrt{e_{1}^{2} \sin ^{4} \theta+4 e_{2}^{2} \cos ^{2} \theta}\right]
$$

while the polarization vector (if the wave vector $\vec{k}$ lies in the plane $\mathrm{x} 0 \mathrm{z}$ ) can be written as:

$$
\vec{e}=\left(1,-i \frac{\varepsilon_{2}}{N^{2}-\varepsilon_{1}}, \frac{N^{2} \sin \theta \cos \theta}{N^{2} \sin ^{2} \theta-\varepsilon_{3}}\right),
$$

where $\cos \theta=k_{2} / k$, and $\varepsilon_{1}, \varepsilon_{2}, \varepsilon_{3}$ are the components of the dielectric permeability tensor.

Let us assume that the hot addition of anisotropic protons has the bi-Maxwellian velocity distribution. Then the expressions for the components of the dielectric permeability tensor have the form /1/:

$$
\begin{gathered}
\varepsilon_{11}=\varepsilon_{22}=\varepsilon_{1}=1+\frac{\left(\omega_{p i}^{c}\right)^{2}}{\omega_{B i}^{2-}}+\frac{A \mu \frac{\left(\omega_{p i}^{c}\right)^{2}}{\omega^{2}}+i(1+A) \mu \frac{\left(\omega_{p i}^{c}\right)^{2}}{\omega \omega_{B i}} \sqrt{\pi} z,}{\varepsilon_{12}=-\varepsilon_{21}=i \varepsilon_{2}=i\left[\frac{\left(\omega_{p i}^{c}\right)^{2} \omega}{\omega_{B i}^{3}}-\mu \frac{\left(\omega_{p i}^{c}\right)^{2}}{\omega \omega_{B i}}-i A \mu \frac{\left(\omega_{p i}^{c}\right)^{2}}{\omega^{2}} \sqrt{\pi} z\right],} \\
\varepsilon_{33}=\varepsilon_{3}=1-\frac{\left(\omega_{p i}^{c}\right)^{2}}{\omega^{2}}-\frac{\left(\omega_{p e}\right)^{2}}{\omega^{2}} .
\end{gathered}
$$

Writing (3)-(5), we used the following notation: $\omega_{p i}^{c}, \omega_{p e}$ are the plasma frequencies of cold protons and electrons; $\omega_{B i}$ is the proton gyrofrequency; $A=\left(T_{\perp}^{p e} / T_{\|}-1\right)$ is the temperature anisotropy of hot protons; $\mu=N_{i h} / N_{i c}$ is the relative concentration of hot protons; $z=\omega_{B i} /\left|k_{2}\right| v_{\|}^{h}, v_{\|}^{h}=\sqrt{2 T_{\|} / m_{i}}$ :

Besides, while deriving (3)-(5), inequalities $z \ll 1$ and $k_{x} v_{\perp}^{h} / \sqrt{2} \omega_{B i} \ll<1$ were assumed to be fulfilled. The latter inequality, as is seen from (3), (4), is unimportant provided that effects of 
collisionless generation-damping are neglected. Note, also, that the second term in (4) is the uncompensated part of the electron contribution, since $N_{i c}+N_{i n}=N_{e}$.

For the longitudinal propagation $(\cos \theta=1)$ the dispersive equation (1) is reduced to the form:

$$
\omega^{2} \approx k^{2} V_{A}^{2}-A \mu \omega_{B i}^{2}(1 \pm i \sqrt{\pi} z) \pm\left(\omega^{2}-\mu \omega_{B i}^{2}\right) \frac{\omega}{\omega_{B i}}
$$

where $V_{A}=B_{0} / \sqrt{4 \pi m_{i} N_{i c}}$ is Alfven velocity.

Let us analyze (6) at a positive anisotropy (let us assume $A \approx 1$ for estimations).

A. $\operatorname{Re} \omega>\sqrt{\mu} \omega_{B i}$. Taking into account the last term in (6) as a small addition (since $\omega<<\omega_{B i}$ ), we have:

$$
\omega=\sqrt{k^{2} V_{A}^{2}-A \mu \omega_{B i}^{2}}\left[1 \pm \frac{k^{2} V_{A}^{2}-(1+A) \mu \omega_{B i}^{2}}{2 \omega_{B i} \sqrt{k^{2} V_{A}^{2}-A \mu \omega_{B i}^{2}}} \pm i A \frac{\mu \omega_{B i}^{2} \sqrt{\pi z}}{2\left(k^{2} \nabla_{A}^{2}-A \mu \omega_{B i}^{2}\right)}\right]
$$

It is seen that with the increase of a wave number, (7) goes over into dispersive dependencies of Aand FMS- waves for cold plasma (taking into account the hot anisotropic addition in imaginary part).

B. $\operatorname{Re} \omega=\sqrt{\mu} \omega_{B i}$. In this case dispersive relation can be written

$$
\omega=\sqrt{\mu} \omega_{B i}\left(1 \pm i A \frac{\sqrt{\pi} z}{2}\right) .
$$

At point $k^{2}=\mu \omega_{B i}^{2}(1+A) / V_{A}^{2}$ the dispersive curves of A- and FMS- waves intersect.

C. $\operatorname{Re} \omega<\sqrt{\mu} \omega_{B i}$. In the region $k^{2} V_{A}^{2}=A \mu \omega_{B i}^{2}$, assuming $\mu>4 A \sqrt{\pi} z$ we have

$$
\omega= \pm \frac{\mu \omega_{B i}}{2}+\frac{\mu \omega_{B i}}{2}\left(1 \pm i A 2 \frac{\sqrt{\pi z}}{\mu}\right) \text {. }
$$

It is seen that the frequency of FMS- waves becomes purely imaginary. At $\mathrm{k}^{2}$ somewhat smaller than $\left(A-\frac{\mu}{2}\right) \frac{\mu \omega_{B i}^{2}}{V_{A}^{2}}$, A- wave as follows from (6), becomes aperiodically
unstable with the increment

$$
\gamma=\sqrt{\mathrm{A} \mu} \omega_{B i} .
$$

Subsituting the obtained solution into (2), it is easy to trace the polarization of each mode. During the longitudinal propagation the polarization along each of the dispersive curves, is conserved (see Figure 1) and is circular ( $L$ or $R$ ). It should be emphasized once again that the obtained modification of MHD oscillations spectrum takes place in a plasma with a sufficiently hot $((z<1))$ anisotropic addition of protons.

At propagation angles other zero, form analyzing (1) and (2), neglecting the imaginary part of the component of the dielectric permeability tensor $\varepsilon_{1}$, we have the following picture. If $\alpha^{2}<\left(\operatorname{Im} \varepsilon_{2}\right)^{2}$, where $\alpha^{2}=\varepsilon_{1}^{2} \sin ^{4} \theta / 4 \cos ^{2} \theta$ then the dispersive curves intersect as before (at point $\operatorname{Re} \omega=\sqrt{\mu} \omega_{B i}$ ) and the direction of the rotation of vector $\vec{E}$ along each of the curves, is conserved. At $\alpha^{2}=\left(\operatorname{Im} \varepsilon_{2}\right)^{2}$ the point of intersection becomes the point of tangency of the dispersive curves crossing which, the left polarization is replaced by the right one and vice versa. A further increase 
of $\alpha^{2}$ leads to the fact that the dispersive curves cease to tough, but while crossing point $\operatorname{Re} \omega=\sqrt{\mu} \omega_{B i}$, the change of polarization is observed as before.

In all the considered regions, only the left-hand polarized waves are generated.

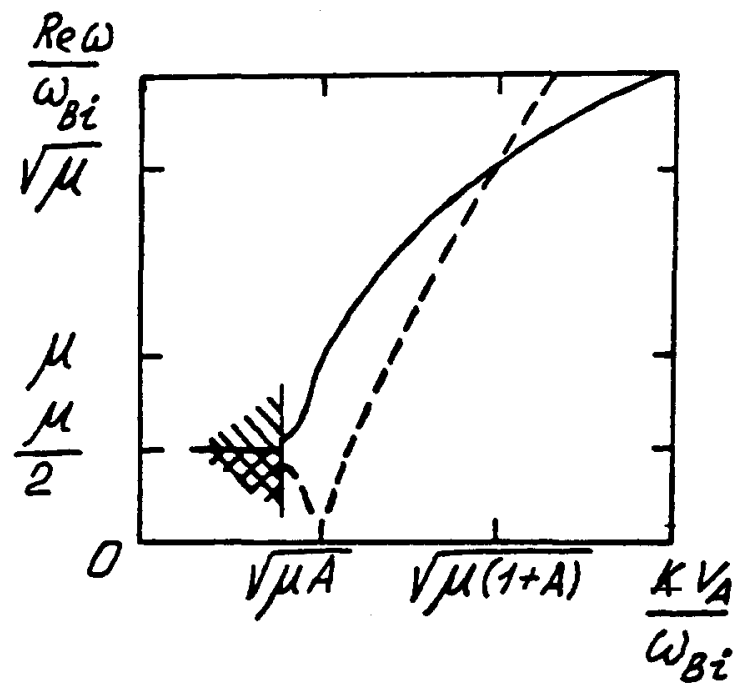

Fig. 1. Schematic dependence of $\operatorname{Re} \omega$ on $k$ during the longitudinal propagation of left-hand $(-)$ and right-hand polarized (---) waves. A>0; instability and damping.

\section{DISCUSSION AND CONCLUSION}

In the previous section we examined the effect of a hot anisotropic addition of protons on the frequency spectra of MHD waves. Taking into account the effect of hot anisropic particles leads, in particular, to the linear transformation of $\mathrm{L}$ waves into right-hand polarized oscillations. The indicated process can be realized in the overlap zone of the magnetospheric ring current and the Earth's plasmasphere according to the following scenario. Left-hand polarized ICW's are generated in the near equatorial zone either during collisionless or hydrodynamic instabilities and propagate into the region of a higher magnetic latitude. If the frequency at which they are generated satisfies the condition $\omega<\sqrt{\mu_{0}} \omega_{B i}$ ( $\mu_{0}$ is the equatorial value of a relative concentration of the hot anisotropic addition), then propagating along the magnetic field lines in the inhomogeneous plasma and reaching the region of transformation, where their frequency becomes equal to the local frequency $\sqrt{\mu} \omega_{B i}$, L-polarized waves are transformed into R-polarized ones.

The proposed mechanism of transformation of L-polarized ICW's into R-polarized ones might probably be useful while interpreting the results of satellite observations of ICW's in the magnetosphere of the Earth and other planets. Particularly, in the cases when the explanation of the presence of the $\mathbf{R}$-waves due to helium ions, runs into difficulties. In any case, the suggested mechanism serves as an addition to the well-known mechanism of transformations in the presence of helium ions.

Besides, hot anisotropic particles lead to strong modification of nonlinear ICW's properties. Matrix elements of interaction of A-oscillations with other types of waves can be modified. Therefore, an inspection is necessary of all the conclusions with respect to decay processes with the participation of A- and FMS- waves in the presence of hot particles with anisotropic temperature in plasma.

In present, we have prepared for publication the paper, devoted to strong nonlinear Alfven waves in plasma with regard to the ring current hot anisotropic protons. The main result of this paper is the 
arising of the electric field due availability of the ring current hot protons.

These results can be useful in the research of waves and particles dynamics in the ring current region during the geomagnetic storms.

Acknowledgments. This work was supported by the NASA Grants NAGW-1619 and NAG5-1500 as well as NSF Grant ATM-9114409.

\section{REFERENCES}

1. A.I. Akheizer (ed.), Plasma Electrodynamics, (in Russian) Nauka, Moscow, 1974.

2. M. Bossen, R.L. McPherron, and C.T. Russel, A statistical study of Pc1 magnetic pulsations at synchronous orbit, J. Geophys. Res., 81, 6063, 1976.

3. J.M. Cornwall, Cyclotron instabilities and electromagnetic emission generation mechanisms, $J$. Geophys. Res., 69, 4515, 1964.

4. B.J. Fraser, Pc-1-2 observations of heavy ion effects by synchronous satellite ATS-6, Planet. Space Sci., 30, 1229, 1982.

5. R. Gendrin, S. Lacourly, A. Roux, J. Solomon, F.Z. Feigin, M. Gokherg, V.A. Troitskaya, and V.L. Yakimenko, Wave packet propagation in an amplifying medium and its application to the dispersion characteristics and to the generation mechanism of Pcl events, Planet. Space Sci., 19, $165,1971$.

6. R. Gendrin, S. Perraut, M. Forgetton, F. Glangeaud, and J.L. Lacoume, ULF waves: Conjugated ground-satellite relationships, Space Sci. Rev., 22, 433, 1978.

7. R. Gendrin, and V.A. Troitskaya, Preliminary results of a micropulsation experiment at conjugate points, Radio Sci., 69D, 1107, 1965.

8. C.F. Kennel, and H.E. Petschek, Limit on stable trapped particle fluxes, J. Geophys. Res., 71, 1, 1966.

9. A. Korth, J. Kremser, S. Perraut, and A. Roux, Interaction of particles with ion cyclotron waves and magnetosonic waves. Observation from GEOS 1 and GEOS 2, Planet. Space Sci., 32, 1393, 1984.

10. J. La Belle, R.A. Treumann, W. Baumjohann, G. Haerendel, N. Sckoplke, and G. Paschmann, The duskside plasmapause/ring current interface: Convection and plasma wave observations, $J$. Geophys. Res., 93, 2573, 1988.

11. E. Leer, K.M. Johansen, and R. Albrigtsen, Group velocity of whistlers in a two-ion plasma., $J$. Geophys. Res., 83, 3125, 1978.

12. B.H. Mauk, C.E. Mcllwain, and R.L. McPherron, Helium cyclotron resonance within the earth's magnetosphere, Geophys. Res. Lett., 8, 103, 1981.

13. B.H. Mauk, and R.L. McPherron, An experimental test of the electromagnetic ion cyclotron instability within the earth's magnetosphere, Phys. Fluids, 23, 2111, 1980.

14. S. Perraut, Wave-particle interactions in the ULF range: GEOS-1 and -2 results, Planet. Space Sci., 30, 1219, 1982.

15. J.L. Rauch, and A. Roux, Ray tracing of ULF waves in a multicomponent magnetospheric 
plasma: Consequences for the generation mechanism of ion cyclotron waves, $J$ Geophys. Res., 87, 8191, 1982.

16. A. Roux, S. Perraut, J.L. Rouch, C. de Villedary, G. Kremser, and A. Korth, Wave-particle interactions near $\boldsymbol{\Omega}_{\mathrm{f}^{+}}$observed on GEOS 1 and 2. 2. Generation of ion-cyclotron waves and heating of $\mathrm{He}^{+}$ions, $\mathrm{He}^{+}$. Geophys. Res., 87, 8174, 1982.

17. D.T. Young, S. Perraut, A. Roux, C. de Villedary, R. Gendrin, A. Kort, G. Kremser, and D. Jones, Wave-particle interactions near observed on GEOS 1 and 2. 1. Propagations of ion cyclotron waves in $\mathrm{He}^{+}$-rich plasma, J. Geophys. Res., 86, 6755, 1981. 